\title{
Statistical Atom: Ionization Energies
}

\author{
Berthold-Georg Englert \\ Sektion Physik, Universität München, Garching, West Germany \\ Z. Naturforsch. 42 a, 825-834 (1987); received February 21, 1987
}

\begin{abstract}
A novel expansion of the Thomas-Fermi potential for a weakly ionized atom is presented. It is employed in calculating the ionization energies of neutral atoms as they are predicted by an extended version of the Thomas-Fermi model which includes the exchange energy and the leading quantum correction. The ionization energy, $I$, can be split into a smooth part, $I_{\text {stat }}$, that is here obtained by the statistical treatment, and an oscillatory part, $I_{\text {osc }}$, which represents significant shell effects. The experimental ionization energies of alkaline atoms are well reproduced by $I_{\text {stat }}$, which approaches the constant of $3.15 \mathrm{eV}$ when the atomic number, $Z$, gets large. It is observed that the amplitude of $I_{\text {osc }}$ is proportional to $Z^{-1 / 3}$, so that shell effects are small compared to the smooth part of $I(Z)$ if $Z$ is sufficiently large (this situation occurs for $Z \gtrsim 10^{3}$, far beyond the Periodic Table). The study of the systematics of $I(Z)$ leads to predictions for the ionization energies of astatine $(Z=85, I \cong 9.5 \mathrm{eV})$ and francium $(Z=87$, $I \cong 3.9 \mathrm{eV}$ ), for which reliable experimental results are not reported.
\end{abstract}

PACS: $31.20 . \mathrm{Lr}, 35.10 . \mathrm{Hn}$

\section{Introduction and Summary}

The nonrelativistic total binding energy of a neutral atom containing $Z$ electrons is composed of a smooth part, $-E_{\text {stat }}(Z, Z)$, and an oscillatory supplement, $-E_{\text {osc }}(Z, Z)$ :

$$
-E(Z, Z)=-E_{\text {stat }}(Z, Z)-E_{\text {osc }}(Z, Z) .
$$

The Thomas-Fermi (TF) statistical model of the atom, together with its quantal refinements, produces [1]

$$
\begin{aligned}
-E_{\text {stat }}(Z, Z)= & 0.768745 Z^{7 / 3}-\frac{1}{2} Z^{2} \\
& +0.269900 Z^{5 / 3},
\end{aligned}
$$

where the first term is the TF binding energy [2], the second one is the correction for strongly bound electrons [3, 4], known as Scott correction, and the third term is due to exchange $[3,5]$ (accounts for 9/11) and quantum corrections to the kinetic energy $[5,6]$ (remaining 2/11). The atomic-binding-energy oscillations that are represented by $E_{\text {osc }}$ have an amplitude proportional to $Z^{4 / 3}$ and are periodic in $Z^{1 / 3}$. For $Z \geqq 22, E_{\text {osc }}$ contributes less than $0.2 \%$ to the binding energy, and less than $0.1 \%$ for $Z \geqq 56$. It has been demonstrated recently by Schwinger and myself [7] that the inclusion of semiclassical quantization into the TF model enables one to understand quantitatively both the amplitude and the

Reprint requests to B.-G. Englert, Sektion Physik, Universität München, Am Coulombwall 1, 8046 Garching. periodicity of $E_{\mathrm{osc}}$. It turns out that this periodicity of $E_{\text {osc }}$ is different from that of the Periodic Table. The latter is determined by the chemical properties of the elements. A related physical quantity is the ionization energy whose periodicity is, indeed, that of the Periodic Table.

The most weakly bound electron is the one that is mainly responsible for the ionization energy of an atom. It is also the one which is least accurately treated in a statistical description. Nevertheless, it is possible to find the smooth part, $I_{\text {stat }}(Z)$, of the ionization energy, $I(Z)$, by employing the refined TF model that produced (2). This $I_{\text {stat }}(Z)$ does, of course, not show any sign of the periodic structure in $I(Z)$, but it represents the first step toward an understanding of the systematics of the Periodic Table.

We denote the TF energy of an atom with nuclear charge $Z$ and $N$ electrons by $E_{\mathrm{TF}}(Z, N)$, and the leading quantum and exchange correction by $\tilde{E}(Z, N)$. The Scott term refers exclusively to the most strongly bound electrons so that it cannot have any significance when ionization is considered. Indeed, the Scott correction is independent of the number of electrons [8]. Thus

$$
E_{\text {stat }}(Z, N)=E_{\mathrm{TF}}(Z, N)+\frac{1}{2} Z^{2}+\tilde{E}(Z, N)
$$

The ionization energy is then

$$
\begin{aligned}
I(Z) & =E(Z, Z-1)-E(Z, Z) \\
& =I_{\mathrm{TF}}(Z)+\tilde{I}(Z)+I_{\mathrm{osc}}(Z),
\end{aligned}
$$

0932-0784 / 87 / 0800-0825 \$01.30/0. - Please order a reprint rather than making your own copy. 


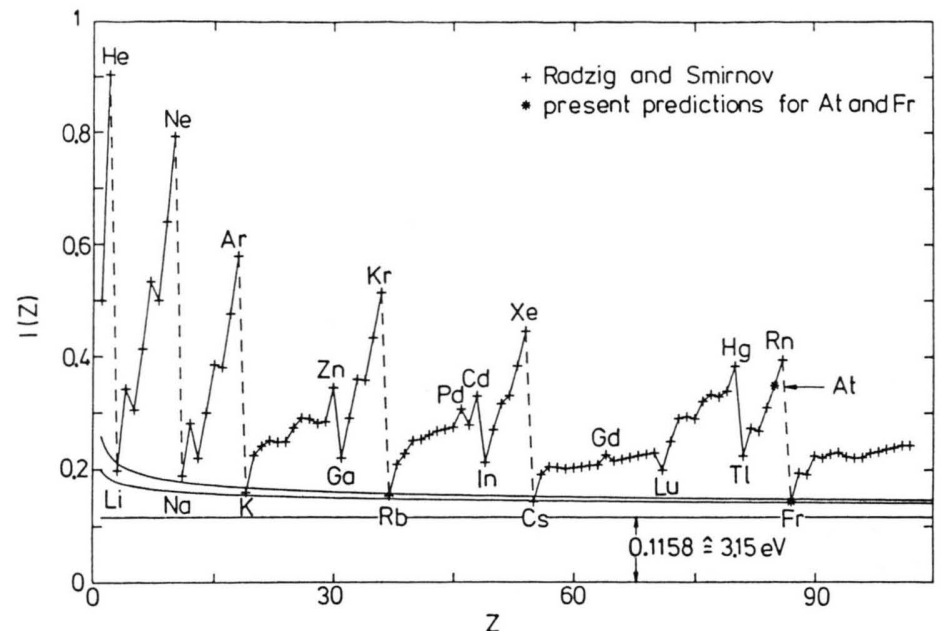

Fig. 1. Experimental ionization energies, supplemented by our predictions for $Z=85$ and 87 , compared with the successive approximations to $I_{\text {stat }}(Z)$ in (9), (smooth curves). The members of each period of the Periodic Table are connected by solid lines, whereas dashed lines guide the eye from inert gases to the respective alkaline metals. where $I_{\mathrm{TF}}$ and $\tilde{I}$ compose the smooth part $I_{\text {stat }}$. With the aid of a novel expansion of the TF potential, one that is suited for investigating the transition from neutral to weakly ionized systems, we demonstrate below that

$$
\begin{aligned}
& E_{\mathrm{TF}}(Z, N)-E_{\mathrm{TF}}(Z, Z) \\
& =0.04731(Z-N)^{7 / 3}\left(1+0.8259 q^{\gamma / 3}\right. \\
& \left.+0.6676 q^{2 \gamma / 3}+\ldots\right)
\end{aligned}
$$

and

$$
\begin{aligned}
& \tilde{E}(Z, N)-\tilde{E}(Z, Z) \\
& =0.06851(Z-N)^{5 / 3}\left(1+0.6290 q^{\gamma / 3}\right. \\
& \left.+0.4142 q^{2 \gamma / 3}+\ldots\right),
\end{aligned}
$$

where

$$
q \equiv 1-N / Z
$$

is the degree of ionization, and the number

$$
\gamma=\frac{1}{2}(\sqrt{73}-7)=0.772002
$$

is familiar from studies of the long-range behavior of the neutral-atom TF potential.

The resulting smooth part of the ionization energy $I(Z)$ is

$$
\begin{aligned}
I_{\text {stat }}(Z)=I_{\mathrm{TF}}(Z)+ & \tilde{I}(Z) \\
=0.1158(1 & +0.7094 Z^{-\gamma / 3} \\
& \left.+0.5177 Z^{-2 \gamma / 3}+\ldots\right) .
\end{aligned}
$$

Its $Z \rightarrow \infty$ limit equals $0.1158 \times 27.21 \mathrm{eV}=3.15 \mathrm{eV}$, of which about $40 \%$ are due to $I_{\mathrm{TF}}$, and $60 \%$ to $\tilde{I}$. This number is significantly different from the one reported recently by Dmitrieva and Plindov [9], namely $4.2 \mathrm{eV}$. These authors' way of handling exchange makes them overestimate what is here called $\tilde{\tilde{I}}$.

In Figure 1, the successive approximations represented by (9) are compared with experimental ionization energies. With the exceptions of astatine $(Z=85)$ and francium $(Z=87)$, these are taken from a recent compilation by Radzig and Smirnov [10]. They do give ionization energies of said elements $(9.0 \mathrm{eV}$ and $4.0 \mathrm{eV}$, respectively), but these numbers do not originate in the analysis of optical spectra, and are therefore considerably less reliable than the rest of the data. For the plot, different numbers were used, obtained by using the systematics of $I(Z)$ for predictions.

Astatine has an electronic configuration in which the last $6 \mathrm{p}$ electron, needed to complete the inertgas configuration of radon, is missing - it is one of the halogens, the heaviest one. The ionization energies of the four other ones - flourine, chlorine, bromine, and iodine - are all about one percent less than the average of the ionization energies of their neighboring elements. There is no reason, why this should not be equally true for astatine. This leads to the predicion $I(Z=85)=9.5 \mathrm{eV}$, which differs significantly from the value of [10].

Francium, the other neighbor or radon, is the heaviest of the alkaline metals. In Figure 1, the other ones - lithium, sodium, potassium, rubidium, and cesium - are all, except for sodium, between the two smooth lines of the linear and the quadratic (in $Z^{-\gamma / 3}$ ) approximations of (9). Francium should 


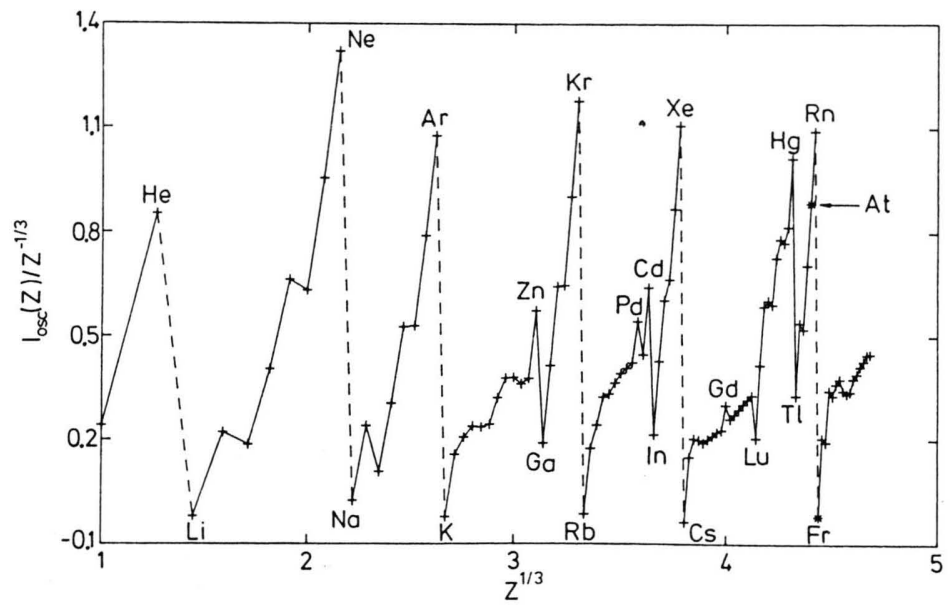

Fig. 2. Experimental values for $I_{\mathrm{osc}}(Z) / Z^{-1 / 3}$ as a function of $Z^{1 / 3}$. As in Fig. 1, our predictions are used for At and Fr, and the members of each period of the Periodic Table are connected by solid lines. also fit into this pattern, so that we predict $I(Z=87)=3.9 \mathrm{eV}$, which differs little from the value of [10].

We observe in Fig. 1 that the $Z \rightarrow \infty$ limit of (9) is consistent with the experimental data, because the oscillatory difference between the experimental ionization energies and $I_{\text {stat }}(Z)$ decreases with increasing atomic number, $Z$. We have already made use of the fact that $I_{\text {stat }}(Z)$ very well reproduces the ionization energies of the alkaline atoms. Could this have been anticipated? Yes, these are the atoms in which the most weakly bound electron does not have partners in its shell, so that shell effects are not expected to be significant. In other words: it is perfectly alright that $I_{\text {stat }}$ accounts only for the (practically constant) "background" in Fig. 1, on top of which we see the oscillations associated with atomic shells. There is certainly no justification for ad-hoc modifications of the theory, aimed at producing an $I_{\text {stat }}$ that somehow interpolates symmetrically through the oscillations of Figure 1. Such was the common procedure in the early days about which Gombás reports in Chap. 22 and Fig. 20 of his textbook [2].

While we are at it, let us take a closer look at the significant shell effects that are contained in the oscillatory part of $I(Z)$,

$$
I_{\text {osc }}(Z)=I(Z)-I_{\text {stat }}(Z) \text {. }
$$

The amplitude of $I_{\text {osc }}$ decreases steadily with growing $Z$, and the period gets longer. The structure of $E_{\text {osc }}$ [Eq. (1)] - amplitude proportional to $Z^{4 / 3}$, period proportional to $Z^{1 / 3}$ - suggests that $I_{\mathrm{osc}}(Z)$ may be of the form $Z^{-1 / 3}$ times an oscillatory function of $Z^{1 / 3}$. This is confirmed to some extent by Fig. 2 where $I_{\text {osc }}(Z) / Z^{-1 / 3}$ is plotted versus $Z^{1 / 3}$. The shell and the sub-shell structure are clearly visible. The overall amplitude of $I_{\text {osc }}$ is about $1.1 Z^{-1 / 3}$, so that

$$
\frac{I_{\text {osc }}(Z)}{I_{\text {stat }}(Z)} \cong 10 Z^{-3}, \quad \text { for large } Z \text {. }
$$

Consequently, shell effects in $I(Z)$ are not small unless $Z \gtrsim 1000$, which is far beyond the Periodic Table. Not that far though, if we remember that $Z^{1 / 3}$ is the relevant parameter; it reaches almost 5 in Figure 2. Therefore, it might be possible to study $I_{\text {osc }}(Z)$ in the asymptotic domain $Z^{1 / 3}>10$, and extrapolate carefully down to $Z^{1 / 3}<5$. Just this was done successfully for $E_{\text {osc }}$ in [7] by including semiclassical quantization into the TF model. I do not see, at this moment, any reason why an analogous calculation for $I_{\text {osc }}$ should fail, although I anticipate the necessity of some refinements.

It is remarkable that (9) performs equally well for both small and large values of $Z$, although it is clearly an approximation which, in the first place, should be reliable only when $Z$ is sufficiently large. A quantitative statement is obtained below [see the remarks after (70)] implying that (9) is not to be trusted for $Z \lesssim 35$. What we witness in Fig. 1 is, once more, the unreasonable quality of a few-term asymptotic expansion.

The main results have now been presented and discussed, so it remains to give a derivation of (5) and (6). This is done in the sequel. The following 
section introduces a new expansion for the $\mathrm{TF}$ potential, which is then employed in computing the numerical values of the coefficients in (5) and (6), and therefore in (9).

\section{New Expansion of the TF Potential}

To set the stage we recall a few basic results of the TF model [2] and draw some immediate conclusions. The TF potential, $V(r)$, is related to the TF function, $f(x)$, through

$$
V(r)+\frac{Z-N}{r_{0}}=-\frac{Z}{r} f(x),
$$

where $r_{0}$ is the radius of the TF atom (beyond which the density of electrons vanishes), and $x$, the $\mathrm{TF}$ measure of distance, is

$$
x=Z^{1 / 3} r / a, a=\frac{1}{2}\left(\frac{3 \pi}{4}\right)^{2 / 3}=0.885341 .
$$

The constant $a$ is such that the differential equation for $f(x)$,

$$
\frac{\mathrm{d}^{2}}{\mathrm{~d} x^{2}} f(x)=\frac{[f(x)]^{3 / 2}}{x^{1 / 2}},
$$

and its boundary conditions $\left(x_{0}=Z^{1 / 3} r_{0} / a\right)$

$$
f(0)=1, f\left(x_{0}\right)=0,-\left.x \frac{\mathrm{d}}{\mathrm{d} x} f(x)\right|_{x_{0}}=q
$$

are free of numerical factors. One observes that $q$, the degree of ionization, determines uniquely both the size of the atom, $x_{0}=x_{0}(q)$, and the TF function $f(x)=f_{\mathrm{q}}(x)$. The TF energy is the $7 / 3$ power of $Z$ times a function of $q$, [11] which we choose to define by

$$
-E_{\mathrm{TF}}(Z, N) \equiv \frac{Z^{7 / 3}}{a} e(q) .
$$

Upon introducing the initial slope $b_{\mathrm{q}}$ of the TF function $f_{\mathrm{q}}(x)$

$$
f_{\mathrm{q}}(x)=1-b_{\mathrm{q}} x+\frac{4}{3} x^{3 / 2}+\ldots,
$$

one can evaluate $e(q)$ by means of

$$
e(q)=\frac{3}{7} b_{q}-\frac{3}{7} \frac{q^{2}}{x_{0}(q)} .
$$

An important connection between $e(q)$ and $x_{0}(q)$ is the differential statement

$$
\frac{\mathrm{d}}{\mathrm{d} q} e(q)=-\frac{q}{x_{0}(q)} \text {. }
$$

The limit $q \rightarrow 0$ in (18) identifies

$$
e(0)=\frac{3}{7} \lim _{q \rightarrow 0} b_{q} \equiv \frac{3}{7} B,
$$

where Baker's [12] constant $B$ is the initial slope of the neutral atom TF function,

$$
F(x)=\lim _{q \rightarrow 0} f_{q}(x) ;=1-B x+\mathscr{O}\left(x^{3 / 2}\right),
$$

The differential equation for $F(x)$ is that of $f_{q}(x)$, (14), subject to the boundary conditions (15), which, for $q=0$, can only be satisfied when $x_{0}=\infty$. Thus, $x_{0}(q=0)=\infty$ and

$$
F(0)=1, F(\infty)=0 .
$$

In conjunction with (14) this implies the large- $x$ form of $F(x)$,

$$
F(x)=\frac{144}{x^{3}}\left[1+\sum_{k=1}^{\infty} c_{\mathrm{k}}\left(\beta x^{-\gamma}\right)^{k}\right],
$$

where $\gamma$ is the number of (8), and the numerical value of the constant $\beta$ is [13], for $c_{1}=-1$,

$$
\beta=13.27097 \text {. }
$$

In contrast to $B$, whose value [13]

$$
B=1.588071
$$

is needed in computing the TF coefficient in (2) [(16) and (20)], the physical significance of $\beta$ has remained obscure until now. We shall see below that it enters the ionization energy.

The coefficients $c_{\mathrm{k}}$ of (23) can be computed recursively, the first ones being

$$
\begin{aligned}
& c_{2}=\frac{201+21 \sqrt{73}}{608}=0.625697, \\
& c_{3}=-\frac{15377+1813 \sqrt{73}}{98496}=-0.313386 .
\end{aligned}
$$

When investigating $q$ dependences it is advantageous to introduce another measure of the strength of the potential, a function $\varphi_{q}(t)$, related to $f_{q}(x)$ 
through [14]

$$
\begin{array}{r}
f_{q}(x)=q \varphi_{q}\left(x / x_{0}(q)\right), \\
\varphi_{q}(t)=\frac{1}{q} f_{q}\left(t x_{0}(q)\right) .
\end{array}
$$

The differential equation obeyed by $\varphi_{q}(t)$ is very similar to (14):

$$
\frac{\mathrm{d}^{2}}{\mathrm{~d} t^{2}} \varphi_{q}(t)=\lambda_{q} \frac{\left[\varphi_{q}(t)\right]^{3 / 2}}{t^{1 / 2}}
$$

where

$$
\lambda_{q} \equiv q^{1 / 2}\left[x_{0}(q)\right]^{3 / 2},
$$

which is going to be an important quantity in the sequel. The boundary conditions on $\varphi_{q}(t)$ are

$\varphi_{q}(0)=\frac{1}{q}, \quad \varphi_{q}(1)=0,\left.\quad \frac{\mathrm{d}}{\mathrm{d} t} \varphi_{q}(t)\right|_{t=1}=-1$.

The limit $q \rightarrow 0$ is not easily performed on the right-hand sides of (27). Nevertheless, it is certainly well-defined, inasmuch as the two left-hand sides approach $F(x)$ and $\Phi(t) \equiv \varphi_{0}(t)$, respectively. This $\Phi(t)$ has been studied in [15]. It obeys

$$
\begin{aligned}
& \frac{\mathrm{d}^{2}}{\mathrm{~d} t^{2}} \Phi(t)=\lambda_{0} \frac{[\Phi(t)]^{3 / 2}}{t^{1 / 2}}, \\
& \Phi(t) \rightarrow \infty, \quad \text { for } t \rightarrow 0, \\
& \Phi(1)=0,\left.\quad \frac{\mathrm{d}}{\mathrm{d} t} \Phi(t)\right|_{t=1}=-1,
\end{aligned}
$$

where [15]

$$
\lambda_{0}=32.7294
$$

is the limiting value of $\lambda_{q}$ as $q \rightarrow 0$.

The physical significance of $\lambda_{0}$ emerges from the observation that (29) has the consequence

$$
x_{0}(q) \cong \lambda_{0}^{2 / 3} q^{-1 / 3}, \text { for } q \gtrsim 0 .
$$

With (19) this implies

$$
e(0)-e(q) \cong \frac{3}{7} \lambda_{0}^{-2 / 3} q^{7 / 3}, \text { for } q \gtrsim 0,
$$

so that $\lambda_{0}$ determines the leading contribution to $I_{\mathrm{TF}}(Z)$ :

$$
\begin{aligned}
I_{\mathrm{TF}}(Z) & =\frac{Z^{7 / 3}}{a}[e(0)-e(q=1 / Z)] ; \\
& =\frac{3}{7 a} \lambda_{0}^{-2 / 3}=0.0473101, \quad \text { for } Z \rightarrow \infty .
\end{aligned}
$$

For small $t, \Phi(t)$ can be expanded in a form analogous to (23),

$$
\Phi(t)=\frac{144 / \lambda_{0}^{2}}{t^{3}}\left[1+\sum_{k=1}^{\infty} d_{k}\left(\alpha t^{\sigma}\right)^{k}\right]
$$

with

$$
\sigma=\frac{1}{2}(\sqrt{73}+7)=\gamma+7, \quad \gamma \sigma=6 .
$$

The constant $\alpha$ has, for $d_{1}=-1$, the numerical value [15]

$$
\alpha=1.04018 ;
$$

like $\beta$ it enters the ionization energy, as we shall see below.

The coefficients $d_{\mathrm{k}}$ in (37) can, just like the $c_{\mathrm{k}}$ of (23), be computed recursively. The two recurrence relations are closely related, one is obtained from the other by interchanging the roles of $\gamma$ and $\sigma$, or equivalently, by changing the sign in front of $\sqrt{7} \overline{3}$, which is also what one has to do in order to get $d_{2}$ and $d_{3}$ from the expressions given for $c_{2}$ and $c_{3}$ in (26).

The analog of (21) is an expansion of $\Phi(t)$ in powers of $(1-t)^{1 / 2}$, whose range of convergence overlaps with that of (37) around $t=0.9$. In [15] highly precise values for $\lambda_{0}$ and $\alpha$ were obtained by matching these two expansions.

The stage is now set to consider a different kind of expansion of the TF potential, i.e., of $f_{q}(x)$ or $\varphi_{q}(t)$, different inasmuch as the expansion parameter is not a measure of distance [as in (17), (23), and (37)], but of the degree of ionization, so that one can study the dependence of various quantities on $q$. For instance, one can count powers of the difference $b_{q}-B$, which is small for $q \gtrsim 0$, as shown in

$$
f_{q}(x)=F(x)-\sum_{k=1}^{\infty}\left(b_{q}-B\right)^{k} f_{k}(x),
$$

where the small- $x$ form of $f_{q}(x)$, Eq. (17), requires

$$
f_{\mathrm{k}}(0)=0, \text { for } k=1,2, \ldots,
$$

and

$$
\left.\frac{\mathrm{d}}{\mathrm{d} x} f_{\mathrm{k}}(x)\right|_{x=0}= \begin{cases}1, & \text { for } k=1, \\ 0, & \text { for } k=2,3, \ldots .\end{cases}
$$

To first order in $b_{q}-B,(40)$ was already considered by Fermi [16]; an investigation of the whole series was performed by Plindov and Dmitrieva [17].

By inserting (40) into the differential equation (14) and identifying powers of $b_{q}-B$, one finds a 
hierarchy of differential equations which (in principle, not in practice) enables one to calculate all $f_{k}(x)$. Since (40) converges rapidly only for sufficiently small values of $x$, it is of little help when investigating ionization energies or other properties of weakly ionized atoms. [Recall that, in view of the $x_{0}(q)$ in (18), the behavior of $f_{q}(x)$ at the edge of the atom is relevant.] Nevertheless, there is a useful lesson to be learned about the functions $f_{k}(x)$.

Let us consider the limit $q \rightarrow 0$ in (27) with $f_{q}(x)$ from (40). First note that

$$
\begin{aligned}
\lim _{q \rightarrow 0} & \frac{1}{q} F\left(t x_{0}(q)\right) \\
= & \left.\frac{144 / \lambda_{q}^{2}}{t^{3}}\left[1+\sum_{k=1}^{\infty} c_{k}\left(\beta t^{-\gamma}\left[x_{0}(q)\right]^{-\gamma}\right)^{k}\right]\right|_{q \rightarrow 0} \\
& =\frac{144 / \lambda_{0}^{2}}{t^{3}}
\end{aligned}
$$

which is the leading term of the expansion (37). Then infer that the $k$-th term in (37) emerges from the $q \rightarrow 0$ limit of the $k$-th term in (40), and conclude that

$$
\begin{gathered}
f_{k}(x) \rightarrow-\frac{144}{x^{3}} d_{k}\left[\frac{\alpha x^{\sigma}}{\lim _{q \rightarrow 0}\left(b_{q}-B\right)\left[x_{0}(q)\right]^{\sigma}}\right]^{k}, \\
\text { for } x \rightarrow \infty .
\end{gathered}
$$

Further, it is straightforward to check that [16]

$$
\begin{aligned}
f_{1}(x)= & {\left[F(x)+\frac{1}{3} x \frac{\mathrm{d}}{\mathrm{d} x} F(x)\right] } \\
& \times \int_{0}^{x} \mathrm{~d} x^{\prime}\left[F\left(x^{\prime}\right)+\frac{1}{3} x^{\prime} \frac{\mathrm{d}}{\mathrm{d} x^{\prime}} F\left(x^{\prime}\right)\right]^{-2} \\
= & \frac{1}{48} \frac{1}{\beta \gamma(\gamma+\sigma)} x^{\sigma-3}\left[1+\mathscr{P}\left(\beta x^{-\gamma}\right)\right]
\end{aligned}
$$

where $\mathscr{P}(y)$ stands for the phrase "powers of $y$ ". This supplies the $k=1$ version of (44) with the consequence

$\lim _{q \rightarrow 0}\left(b_{q}-B\right)\left[x_{0}(q)\right]^{\sigma}=\frac{(12)^{4}}{3} \alpha \beta \gamma(\gamma+\sigma)$,

so that the large- $x$ form of $f_{k}(x)$ is

$$
\begin{aligned}
& f_{k}(x)= \\
& \quad-\frac{144}{x^{3}} d_{k}\left[\frac{3}{(12)^{4}} \frac{1}{\beta \gamma(\gamma+\sigma)} x^{\sigma}\right]^{k}\left[1+\mathscr{P}\left(\beta x^{-\gamma}\right)\right] .
\end{aligned}
$$

This equation can also be derived by investigating the recurrence relation of the $f_{k}(x)$. Some additional effort is then required to show that the $d_{k}$ 's of (47) are identical with the ones of (37).

Upon introducing $h_{q}$ by means of

$$
b_{q}-B=\frac{(12)^{4}}{3} \alpha \beta \gamma(\gamma+\sigma)\left[h_{q} / x_{0}(q)\right]^{\sigma}
$$

into (47) and then (40), followed by making the "powers of $\beta x^{-\gamma}$ " explicit, we arrive at

$$
\begin{aligned}
f_{q}(x)=q\left(\frac{\lambda_{0}}{\lambda_{q}}\right)^{2} h_{q}^{3} & {\left[\Phi\left(h_{q} x / x_{0}(q)\right)\right.} \\
& \left.+\sum_{k=1}^{\infty} c_{k}\left[\beta x^{-\gamma}\right]^{k} \psi_{k}\left(h_{q} x / x_{0}(q)\right)\right] .
\end{aligned}
$$

This powerful new expansion of the TF potential, here stated for $f_{q}(x)$, converges fast for $x \cong x_{0}(q)$ in contrast to the series (40), which is rapidly convergent for $x \ll x_{0}(q)$. The neutral-atom TF function $F(x)$ must be recovered in the limit $q \rightarrow 0$, which, in view of the coefficients $c_{\mathrm{k}}$ in (49), implies the small- $t$ behavior

$$
\psi_{k}(t)=\frac{144 / \lambda_{0}^{2}}{t^{3}}\left[1+\mathscr{P}\left(\alpha t^{\sigma}\right)\right] .
$$

For the subsequent applications of (49) it is necessary to study the first $\psi_{k}$ 's in some detail. The insertion of (49) into the differential equation obeyed by $f_{q}(x),(14)$, produces

$$
\left[\frac{\mathrm{d}^{2}}{\mathrm{~d} t^{2}}-\frac{3}{2} \lambda_{0} \sqrt{\Phi(t) / t}\right] t^{-\gamma} \psi_{1}(t)=0
$$

and

$$
\begin{aligned}
{\left[\frac{\mathrm{d}^{2}}{\mathrm{~d} t^{2}}-\frac{3}{2} \lambda_{0}\right.} & \sqrt{\Phi(t) / t}] t^{-2 \gamma} \psi_{2}(t) \\
& =\frac{3 / 8}{c_{2}} \frac{\lambda_{0}}{\sqrt{t \Phi(t)}}\left[t^{-\gamma} \psi_{1}(t)\right]^{2},
\end{aligned}
$$

when terms linear and quadratic in $\beta x^{-\gamma}$ are identified. It is simple to check that

$$
\psi_{0}(t)=t^{\gamma} \frac{1}{3 t^{2}} \frac{\mathrm{d}}{\mathrm{d} t}\left[t^{3} \Phi(t)\right]
$$

is one solution of (51). Its Wronskian with $\psi_{1}(t)$ is

$$
\begin{aligned}
\psi_{0}(t) \frac{\mathrm{d}}{\mathrm{d} t} \psi_{1}(t) & -\psi_{1}(t) \frac{\mathrm{d}}{\mathrm{d} t} \psi_{0}(t) \\
& =\frac{1}{3}\left(\frac{12}{\lambda_{0}}\right)^{4} \alpha \sigma(\gamma+\sigma) t^{2 \gamma} .
\end{aligned}
$$


It is possible to express $\psi_{1}(t)$ as a certain integral involving $\psi_{0}(t)$, in analogy to (45), but this is not really helpful. It is much easier to expand $\psi_{1}(t)$ in powers of $\alpha t^{\sigma}$ and $\sqrt{1-t}$, and match the two expansions, following the procedure of [15]. This way one obtains

$$
\psi_{1}(1)=0.321686353717,
$$

which illustrates once more the high precision of the algorithm. The Wronskian (54) is employed in finding

$$
\begin{aligned}
\psi_{1}^{\prime}(1) \equiv & \left.\frac{d}{\mathrm{~d} t} \psi_{1}(t)\right|_{t=1}=(4+\gamma) \psi_{1}(1) \\
& -\left(\frac{12}{\lambda_{0}}\right)^{4} \alpha \sigma(\gamma+\sigma)=0.286916,
\end{aligned}
$$

whereas the differential equation (51) supplies

$$
\begin{aligned}
\left.\psi_{1}^{\prime \prime}(1) \equiv \frac{\mathrm{d}^{2}}{\mathrm{~d} t^{2}} \psi_{1}(t)\right|_{t=1} & =2 \gamma \psi_{1}^{\prime}(1)-\gamma(\gamma+1) \psi_{1}(1) \\
& =0.00293603 .
\end{aligned}
$$

This kind of computation can be repeated, with the necessary changes, for $\psi_{2}(t)$, with the results

$$
\begin{aligned}
& \psi_{2}(1)=-0.000155492, \\
& \left.\psi_{2}^{\prime}(1) \equiv \frac{\mathrm{d}}{\mathrm{d} t} \psi_{2}(t)\right|_{t=1}=-0.00958861 .
\end{aligned}
$$

\section{Calculation of $E_{\mathrm{TF}}(Z, N)$}

We are now prepared to evaluate $e(q)$ for small $q$. The boundary conditions on $f_{q}(x)$ at $x=x_{0}(q)$, expressed in terms of the new expansion (49), read

and

$$
\Phi\left(h_{q}\right)+\sum_{k=1}^{\infty} c_{k}\left(\beta x_{0}^{-\gamma}\right)^{k} \psi_{k}\left(h_{q}\right)=0
$$

$$
\begin{aligned}
\left(\frac{\lambda_{q}}{\lambda_{0}}\right)^{2} & =h_{q}^{3}\left[-h_{q} \frac{\mathrm{d}}{\mathrm{d} h_{q}} \Phi\left(h_{q}\right)\right. \\
& \left.+\sum_{k=1}^{\infty} c_{k}\left(\beta x_{0}^{-\gamma}\right)^{k}\left(\gamma k \psi_{k}\left(h_{q}\right)-h_{q} q \frac{\mathrm{d}}{\mathrm{d} h_{q}} \psi_{k}\left(h_{q}\right)\right)\right] .
\end{aligned}
$$

For small $q, \beta\left[x_{0}(q)\right]^{-\gamma}$ is a small quantity, so that (59) supplies an expansion of $h_{q}$ in powers of $x_{0}^{-\gamma}$.
Inserted into (60), a corresponding expansion is obtained for $\lambda_{q}$. Now, recall that $x_{0}(q)$ and $\lambda_{q}$ are related through (29), i.e., $x_{0}^{-\gamma}=\lambda_{q}^{-2 \gamma / 3} q^{\gamma / 3}$. Consequently, the expansion of $\lambda_{q}$ in powers of $x_{0}^{-\gamma}$ can be converted into one in powers of $q^{\gamma / 3}$. Used in (29) and (19), this leads to a corresponding expansion of $e(q)$ :

$e(q)=e(0)-\frac{3}{7} \lambda_{0}^{-2 / 3} q^{7 / 3}\left[1+\sum_{k=1}^{\infty} e_{k} q^{k \gamma / 3}\right]$,

where one recognizes the leading term, found earlier in Eq. (34).

The constants $e_{1}, e_{2}, \ldots$ of (61) can, indeed, be obtained this way. It is, however, somewhat easier to make use of a few of the relations given above, instead of explicitly employing (60). For instance, (19), (61), and (29) can be combined into

$$
\left(\lambda_{q} / \lambda_{0}\right)^{-2 / 3}=1+\sum_{k=1}^{\infty}\left(1+\frac{k \gamma}{7}\right) e_{k} q^{k \gamma / 3} .
$$

Upon utilizing (18), (20), and (48) as well, the identity

$$
\begin{aligned}
\sum_{k=1}^{\infty} k e_{k} q^{(k-1) \gamma / 3} & \\
= & \frac{7}{3}\left(\frac{12}{\lambda_{0}}\right)^{4} \lambda_{0}^{-2 \gamma / 3} \alpha \beta(\gamma+\sigma)\left[h_{q}\left(\lambda_{q} / \lambda_{0}\right)^{-2 / 3}\right]^{\sigma}
\end{aligned}
$$

can be established. Here, the limit $q \rightarrow 0$ immediately identifies

$$
\begin{aligned}
e_{1} & =\frac{7}{3}\left(\frac{12}{\lambda_{0}}\right)^{4} \lambda_{0}^{-2 \gamma / 3} \alpha \beta(\gamma+\sigma) \\
& =0.825908,
\end{aligned}
$$

which, as promised above, reveals the physical significance of the constants $\alpha$ and $\beta$.

To first order in $x_{0}^{-\gamma},(59)$ says

$$
\begin{aligned}
h_{q} & =1-\psi_{1}(1) \beta\left[x_{0}(q)\right]^{-\gamma}+\ldots \\
& =1-\beta \lambda_{0}^{-2 \gamma / 3} \psi_{1}(1) q^{\gamma / 3}+\mathscr{O}\left(q^{2 \gamma / 3}\right),
\end{aligned}
$$

so that, in conjunction with (62), the order $q^{\gamma / 3}$ of (63) supplies

$$
\begin{aligned}
e_{2} & =\frac{1}{2} e_{1} \sigma\left[\left(1+\frac{\gamma}{7}\right) e_{1}-\beta \lambda_{0}^{-2 \gamma / 3} \psi_{1}(1)\right] \\
& =0.667554 .
\end{aligned}
$$




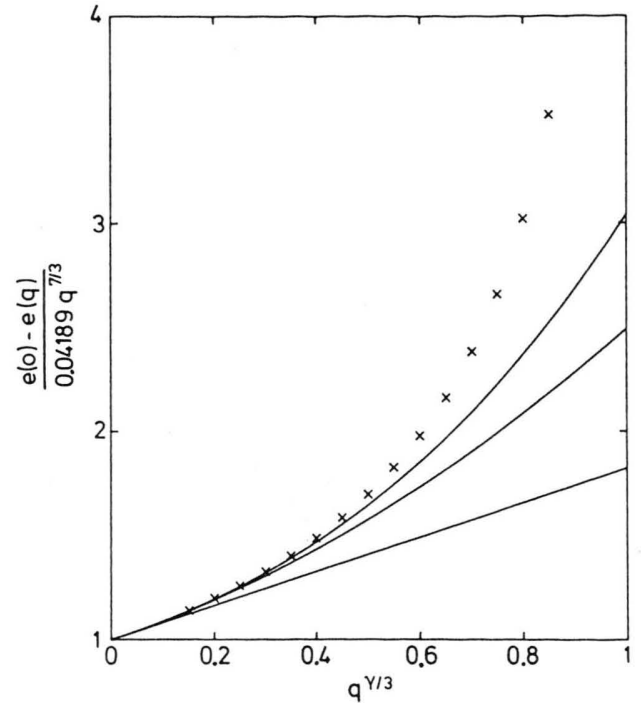

Fig. 3. Successive approximations of (70) compared with actual data (crosses).

Naturally, the subsequent coefficients in (61) can be computed analogously. We report what emerges for $e_{3}$ :

$$
\begin{aligned}
e_{3}= & \frac{6-\gamma}{9} \frac{e_{2}^{2}}{e_{1}}+\frac{8+3 \sigma}{7} e_{1} e_{2}-\frac{78+97 \sigma}{147} e_{1}^{3} \\
& +\frac{\sigma}{3}\left[\psi_{1}(1) \psi_{1}^{\prime}(1)+c_{2} \psi_{2}(1)\right] \beta^{2} \lambda_{0}^{-2 \gamma / 3} e_{1} \\
= & 0.550086 .
\end{aligned}
$$

So we have, indeed, found the coefficients that, together with the over-all factor of (36), produce the $E_{\mathrm{TF}}(Z, N)$ reported earlier in (5).

The method of [17], which is related to the one presented here [it is based upon (40) instead of (49)], gave

$$
\frac{\sigma}{7} e_{1}=0.9102
$$

and

$$
\frac{\sigma+\gamma}{7} e_{2}=0.8246
$$

which are in error by $-0.74 \%$ and $1.2 \%$, respectively. No value for $e_{3}$ is reported there, which is no surprise, because that method cannot produce it correctly. Expressed in our language, in [17] the values of $\lambda_{0}, \alpha, \psi_{1}(1), \psi_{2}(1), \ldots$ are obtained by using the expansions of $\Phi(t), \psi_{1}(t), \psi_{2}(t), \ldots$ in powers of $\alpha t^{\sigma}$ for $t=1$, where they do not converge. As far as $\Phi(t)$ and $\psi_{1}(t)$ are concerned, no significant error is caused [for instance, summing 40 terms gives $\psi_{1}(1)=0.32168796$ instead of the number of (55)], but $\psi_{2}(1)$ and $\psi_{2}^{\prime}(1)$ are seriously affected $[-0.00224014$ and -0.355000 , respectively, instead of (58), and summing more than 40 terms makes it, rapidly, even worse], and the same is true for $\psi_{3}, \psi_{4}, \ldots$.

The successive approximations that are represented by

$$
\begin{aligned}
\frac{e(0)-e(q)}{0.04189 q^{7 / 3}} \cong 1 & +0.8259 q^{\gamma / 3} \\
& +0.6676 q^{2 \gamma / 3}+0.5501 q^{3 \gamma / 3}
\end{aligned}
$$

are compared with actual data in Figure 3. We observe that the linear approximation is practically useless, the quadratic one can be used for $q^{\gamma / 3} \lesssim 0.4$, the cubic one for $q^{\gamma / 3} \lesssim 0.5$. For $q=1 / Z$, this requires $Z \gtrsim 35$, and $Z \gtrsim 15$, respectively.

\section{Calculation of $\tilde{E}(Z, N)$}

According to Schwinger [5] the sum of the exchange energy and the leading quantum correction to the kinetic energy is given by

$$
\begin{aligned}
\tilde{E}(Z, N) & =-\frac{11}{32 a^{2}} Z^{5 / 3} \int_{0}^{x_{0}(q)} \mathrm{d} x\left[f_{q}(x)\right]^{2} \\
& \equiv-\frac{11}{32 a^{2}} Z^{5 / 3} \tilde{e}(q),
\end{aligned}
$$

which is the result of the perturbative evaluation of more involved expressions. For detail consult [5] or [18]. The numerical value of the neutral-atom integral is $\tilde{e}(0)=0.615435$, it gives the $Z^{5 / 3}$ term in (2).

The difference

$$
\begin{aligned}
\tilde{e}(0)-\tilde{e}(q)= & \int_{x_{0}(q)}^{\infty} \mathrm{d} x[F(x)]^{2} \\
& +\int_{0}^{x_{0}(q)} \mathrm{d} x\left([F(x)]^{2}-\left[f_{q}(x)\right]^{2}\right)
\end{aligned}
$$


can be expanded in powers of $\beta\left[x_{0}(q)\right]^{-\gamma}$, after (23) and (49) are used to rewrite the integrands. The outcome is

$$
\begin{aligned}
\tilde{e}(0)-\tilde{e}(q)= & \frac{(12)^{4}}{\left[x_{0}(q)\right]^{5}}\left\{\left[\frac{1}{5}+h_{q}^{5} \int_{0}^{h_{q}} \frac{\mathrm{d} t}{t^{6}}\left(1-\left(\frac{\lambda_{0}}{12}\right)^{4} t^{6}[\Phi(t)]^{2}\right)\right]\right. \\
& +2 \sum_{k=1}^{\infty} c_{k}\left(\beta x_{0}^{-\gamma}\right)^{k}\left[\frac{1}{5+k \gamma}+h_{q}^{5+k \gamma} \int_{0}^{h_{q}} \frac{\mathrm{d} t}{t^{6+k \gamma}}\left(1-\left(\frac{\lambda_{0}}{12}\right)^{4} t^{6} \Phi(t) \psi_{k}(t)\right)\right] \\
& +\sum_{j, k=1}^{\infty} c_{j} c_{k}\left(\beta x_{0}^{-\gamma}\right)^{j+k}\left[\frac{1}{5+(j+k) \gamma}+h_{q}^{5+(j+k) \gamma} \int_{0}^{h_{q}} \frac{\mathrm{d} t}{t^{6+(j+k) \gamma}}\right. \\
& \left.\left.\times\left(1-\left(\frac{\lambda_{0}}{12}\right)^{4} t^{6} \psi_{j}(t) \psi_{k}(t)\right)\right]\right\} .
\end{aligned}
$$

When now powers of $q^{\gamma / 3}$ are identified, the integrals

$$
\begin{aligned}
J_{0} & =\frac{1}{5}+\int_{0}^{1} \frac{\mathrm{d} t}{t^{6}}\left(1-\left(\frac{\lambda_{0}}{12}\right)^{4} t^{6}[\Phi(t)]^{2}\right) \\
& =0.844849, \\
J_{1} & =\frac{1}{5+\gamma}+\int_{0}^{1} \frac{\mathrm{d} t}{t^{6+\gamma}}\left(1-\left(\frac{\lambda_{0}}{12}\right)^{4} t^{6} \Phi(t) \psi_{1}(t)\right) \\
& =0.0787772, \\
J_{11} & =\frac{1}{5+2 \gamma}+\int_{0}^{1} \frac{\mathrm{d} t}{t^{6+2 \gamma}}\left(1-\left(\frac{\lambda_{0}}{12}\right)^{4} t^{6}\left[\psi_{1}(t)\right]^{2}\right) \\
& =-2.59442, \\
J_{2} & =\frac{1}{5+2 \gamma}+\int_{0}^{1} \frac{\mathrm{d} t}{t^{6+2 \gamma}}\left(1-\left(\frac{\lambda_{0}}{12}\right)^{4} t^{6} \Phi(t) \psi_{2}(t)\right) \\
& =2.06237
\end{aligned}
$$

show up. The result is

$$
\begin{aligned}
& \tilde{e}(0)-\tilde{e}(q) \\
& =\left(\frac{12}{\lambda_{0}}\right)^{4} \lambda_{0}^{2 / 3} J_{0} q^{5 / 3}\left(1+\tilde{e}_{1} q^{\gamma / 3}+\tilde{e}_{2} q^{2 \gamma / 3}+\ldots\right),
\end{aligned}
$$

where the factor in front equals 0.156210 , and $\tilde{e}_{1}$ and $\tilde{e}_{2}$ are given by

and

$$
\tilde{e}_{1}=\frac{5}{3} \gamma \frac{e_{2}}{e_{1}}-2 \beta \lambda_{0}^{-2 \gamma / 3} \frac{J_{1}}{J_{0}}=0.628951
$$

$$
\begin{aligned}
\tilde{e}_{2}= & \frac{5}{2} \gamma \frac{e_{3}}{e_{1}}-\frac{15}{2}(3 \gamma-2)\left(\frac{e_{2}}{e_{1}}\right)^{2} \\
& +\frac{2}{3}(3-\gamma) \frac{e_{2} \tilde{e}_{1}}{e_{1}}+\beta^{2} \lambda_{0}^{-4 \gamma / 3} \frac{J_{11}+2 c_{2} J_{2}}{J_{0}} \\
= & 0.414174 .
\end{aligned}
$$

* Note added in proof: After completion of this work H. T. Duong et al., Europhys. Lett. 3, 175 (1987) reported spectroscopic measurements on francium from which they could extract the ionization energy. Depending on the finer details of the data, values between 4.03 and $4.12 \mathrm{eV}$ were obtained. These agree favorably with our prediction.
These, combined with the factor of (71), are the coefficients of (6).

Please note that $\tilde{I}(Z)=\tilde{E}(Z, Z-1)-\tilde{E}(Z, Z)$ is of the same size as $I_{\mathrm{TF}}(Z)=E_{\mathrm{TF}}(Z, Z-1)$ given degree of ionization, i.e., for a given ratio $N / Z$, is smaller than $E_{\mathrm{TF}}(Z, N)$ by a factor $Z^{-2 / 3}$. The reason is, of course, that $I_{\mathrm{TF}}$ and $\tilde{I}$ are determined by the differences $E_{\mathrm{TF}}(Z, N)-E_{\mathrm{TF}}(Z, Z)$ and $\tilde{E}(Z, N)-\tilde{E}(Z, Z)$, which are proportional to $(Z-N)^{7 / 3}$ and $(Z-N)^{5 / 3}$, respectively, and of comparable magnitude when $Z-N=1$.*

\section{Acknowledgement}

I would like to say thank you to the LeibnizRechenzentrum der Bayerischen Akademie der Wissenschaften for making computer time available to me. - $E_{\mathrm{TF}}(Z, Z)$ in contrast to $\tilde{E}(Z, N)$, which, for a 
[1] Atomic units are used, unless stated otherwise. Distances are multiples of the Bohr radius $\hbar^{2} /\left(m e^{2}\right)$ $=0.5292 \AA$, energies multiples of twice the Rydberg constant $m e^{4} / \hbar^{2}=27.21 \mathrm{eV}$.

[2] See, for example, P. Gombás, die statistische Theorie des Atoms und ihre Anwendungen, Springer, Wien 1949.

[3] J. M. C. Scott, Philos. Mag. 43, 859 (1952).

[4] J. Schwinger, Phys. Rev. A 22, 1827 (1980).

[5] J. Schwinger, Phys. Rev. A 24, 2353 (1981).

[6] See also G. I. Plindov and I. K. Dmitrieva, Phys. Lett. 64 A, 348 (1978), where (2) occurs for the first time; this derivation of the $Z^{5 / 3}$ term is hardly satisfactory, however, because it involves arbitrary cut-offs of the density for small and large distances. Also. the Scott term was not obtained correctly.

[7] B.-G. Englert and J. Schwinger, Phys. Rev. A 32, 47 (1985).

[8] This property of the Scott term is stated explicitly by B.-G. Englert and J. Schwinger, Phys. Rev. A 32, 36 (1985). It is implicit in earlier derivations.

[9] I. K. Dmitrieva and G. I. Plindov, J. Phys. Paris 45, 85 (1984).

[10] A. A. Radzig and B. M. Smirnov, Reference Data on Atoms, Molecules, and Ions, Springer-Verlag, Berlin 1985, Springer Series in Chemical Physics, Vol. 31.
[11] For a derivation of this and many related statements, based upon the scaling properties of the TF energy functional, consult B.-G. Englert and J. Schwinger, Phys. Rev. A 29, 2331 (1984).

[12] E. B. Baker, Phys. Rev. 36, 630 (1930).

[13] S. Kobayashi, T. Matsukama, S. Nagai, and K. Umeda, J. Phys. Soc. Japan 10, 759 (1955). In this paper something went wrong with the recursive calculation of $c_{1}, c_{2}, \ldots$. The tabulated values for $c_{1}, \ldots, c_{10}$ are correct, whereas $c_{11}, \ldots, c_{17}$ are false (with increasing error). This did, however, not affect the results as far as the values of $B$ and $\beta$, given in the Abstract, are concerned.

[14] G. I. Plindov and I. K. Dmitrieva, Dokl. Akad. Nauk BSSR 19, $788(1975)$, where (28)-(30) are used as the basis for a calculation of $E_{\mathrm{TF}}(Z, N)$ for $N \ll Z$

[15] B.-G. Englert, Phys. Rev. A 33, 2146 (1986).

(16] E. Fermi, Mem. Accad. d'Italia 1, 149 (1930).

[17] G. I. Plindov and I. K. Dmitrieva, Dokl. Akad. Nauk BSSR 21, 209 (1977)

[18] B.-G. Englert and J. Schwinger, Phys. Rev. A 29, 2339 (1984). 